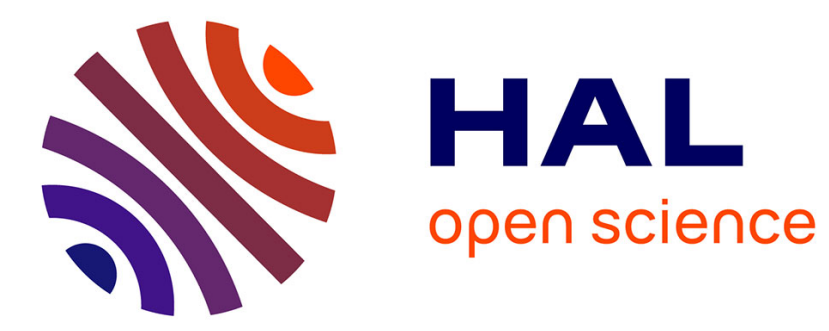

\title{
Phase Estimation in Differential-Interference-Contrast (DIC) Microscopy
}

Lola Bautista, Simone Rebegoldi, Laure Blanc-Féraud, Marco Prato, Luca

Zanni, Arturo Plata

\section{To cite this version:}

Lola Bautista, Simone Rebegoldi, Laure Blanc-Féraud, Marco Prato, Luca Zanni, et al.. Phase Estimation in Differential-Interference-Contrast (DIC) Microscopy. IEEE International Symposium on Biomedical Imaging (ISBI), Apr 2016, Prague, Czech Republic. pp.136-139. hal-01255004

\section{HAL Id: hal-01255004 \\ https://hal.inria.fr/hal-01255004}

Submitted on 14 Jan 2016

HAL is a multi-disciplinary open access archive for the deposit and dissemination of scientific research documents, whether they are published or not. The documents may come from teaching and research institutions in France or abroad, or from public or private research centers.
L'archive ouverte pluridisciplinaire HAL, est destinée au dépôt et à la diffusion de documents scientifiques de niveau recherche, publiés ou non, émanant des établissements d'enseignement et de recherche français ou étrangers, des laboratoires publics ou privés. 


\title{
PHASE ESTIMATION IN DIFFERENTIAL-INTERFERENCE-CONTRAST (DIC) MICROSCOPY
}

\author{
Lola Bautista $^{\star \dagger}$, Simone Rebegoldi ${ }^{\S}$,Laure Blanc-Féraud ${ }^{\dagger}$, Marco Prato $^{\S}$, Luca Zanni $^{\S}$, Arturo Plata \\ * Escuela de Ingeniería de Sistemas y Escuela de Física, Universidad Industrial de Santander, \\ 680002 Bucaramanga, Colombia \\ ${ }^{\dagger}$ CNRS, Laboratoire I3S, UMR 7271, Université Nice Sophia Antipolis, \\ 06903 Sophia Antipolis, France \\ $\S$ Dipartimento di Scienze Fisiche, Informatiche e Matematiche, Università di Modena e Reggio Emilia, \\ Via Campi 213/b, 41125 Modena, Italy
}

\begin{abstract}
We present a gradient-based optimization method for the estimation of a specimen's phase function from polychromatic DIC images. The method minimizes the sum of a nonlinear least-squares discrepancy measure and a smooth approximation of the total variation. A new formulation of the gradient and a recent updating rule for the choice of the step size are both exploited to reduce computational time. Numerical simulations on two computer-generated objects show significant improvements, both in efficiency and accuracy, with respect to a more standard choice of the step size.
\end{abstract}

Index Terms - DIC microscopy, phase estimation, inverse problems, gradient-descent method

\section{INTRODUCTION}

Differential-interference-contrast (DIC) microscopy is an optical microscopy technique designed mainly for biological purposes, to observe unstained transparent specimens under a transmitted-light configuration. Among its advantages are the ability to produce high contrast images and to achieve high lateral resolution since it is possible to use full numerical apertures in the objective [1]. DIC imaging is accomplished from the interference of two waves that have a lateral differential displacement (shear) and are phase shifted relative one to each other. The resulting intensity image is given by a nonlinear function of approximately the spatial gradient of a specimen's optical path length along the direction of shear [2]; this provides a shadow-cast effect in the acquired image which indicates the sign and slope of phase gradients in the specimen [3].

In order to retrieve information hidden in the phase gradients, it is necessary to solve the nonlinear, ill-posed inverse problem of phase estimation from the DIC intensity images.

This work has been supported by the ECOS-Nord grant C15M01, the Universidad Industrial de Santander and the Italian GNCS - INdAM.
Different approaches have been followed in previous work, such as the Phase Shifting algorithm used by Lin et al. in [4] and the method based on the transport-of-intensity equation exploited by Bostan et al. in [5]. In this paper we have followed the approach of rotational-diversity proposed by Preza in [6], which provides information about the specimen-phase function derivative in multiple directions and, by exploiting a least-squares approach, gives the phase estimation as a regularized solution of a smooth nonconvex optimization problem. We propose to regularize the least-squares optimization problem by using a smooth total variation term and we introduce an efficient gradient-descent method for solving the regularized problem. We have added the polychromatic acquisition in order to further increase phase diversity. Our main contributions consist in: i) the extension of the model from grayscale to color images; ii) a novel formulation of the gradient which allows faster computations at each iteration; iii) a clever choice of the step size parameter for the gradientdescent method to improve the speed of convergence [7].

The paper is organized as follows: in section 2 the rotational-diversity polychromatic image formation model and the gradient-based phase estimation method are described. In section 3 the results obtained from simulations of synthetic objects are discussed. Conclusions and future work are presented in section 4 .

\section{MODEL AND METHOD}

\subsection{Polychromatic rotational-diversity model}

In the rotational-diversity model [6], the specimen rotation is modeled as a rotation of the PSF by an angle $\tau_{k}$, the angle that the shear direction makes with the horizontal axis. Using a discrete version of the continuous model presented in [6], the $k$-th diversity intensity image $i_{k, \lambda}(x)$ is described by

$$
i_{k, \lambda}(x)=a_{1}\left|\sum_{x_{0} \in \chi} f\left(x_{0}\right) h_{k, \lambda}\left(x-x_{0}\right)\right|^{2},
$$


where $a_{1}$ is a constant which corresponds to closing the condenser aperture down to a single point; $k \in\{0, \ldots, K-1\}$; $\lambda \in\left\{\lambda_{1}=0.65, \lambda_{2}=0.55, \lambda_{3}=0.45\right\}$ are the values for the RGB wavelengths; $x$ and $x_{0}$ take values on the set $\chi=\{0,1, \ldots, N-1\}^{2}$, being $N$ the number of elements in each dimension of the object and image space; $f\left(x_{0}\right)=e^{-j \frac{\phi\left(x_{0}\right)}{\lambda}}$ is the specimen's transmission function, being $\phi\left(x_{0}\right)$ the specimen's phase function we want to retrieve; $h_{k, \lambda}$ is the $k$-th rotation of the polychromatic DIC amplitude point spread function

$$
h_{k, \lambda}(x)=\frac{1}{2}\left[e^{-j \Delta \theta} p_{k, \lambda}(x-\Delta x, y)-e^{j \Delta \theta} p_{k, \lambda}(x+\Delta x, y)\right],
$$

expressed in terms of the coherent PSF of the microscope's objectives lens $p_{k, \lambda}(x)$ at angle $\tau_{k}$ and for the wavelength $\lambda$, where $2 \Delta \theta$ is the DIC bias retardation and $2 \Delta x$ is the shear.

\subsection{Gradient-based phase estimation}

In order to estimate the phase function $\phi(x)$, we propose to solve the following regularized least-squares problem

$$
\min _{\phi \in \mathbb{R}^{N^{2}}} J(\phi) \equiv \sum_{\lambda=\lambda_{1}}^{\lambda_{3}} \sum_{k=0}^{K-1} \sum_{x \in \chi}\left[o_{k, \lambda}(x)-i_{k, \lambda}(x)\right]^{2}+\mu J_{T V}(\phi),
$$

where $o_{k, \lambda}$ is the $k$-th observed polychromatic image, $i_{k, \lambda}$ is the $k$-th theoretical polychromatic image, $J_{T V}$ is a smooth approximation of the total variation, also known in literature as the hypersurface potential [8] and $\mu$ is the regularization parameter. Problem (3) has been approached by using a gradient-descent method defined as

$$
\phi^{(m+1)}=\phi^{(m)}-\alpha_{m} \nabla J\left(\phi^{(m)}\right),
$$

where $\alpha_{m}$ is the step size and the components of the gradient are given by

$$
\frac{\partial J(\phi)}{\partial \phi\left(x_{0}\right)}=\sum_{\lambda=\lambda_{1}}^{\lambda_{3}} \sum_{k=0}^{K-1}-\frac{4}{\lambda} \cdot \mathcal{I}\left\{\xi\left(\phi, x_{0}, h_{k, \lambda}\right)\right\}+\mu \frac{\partial J_{T V}(\phi)}{\partial \phi\left(x_{0}\right)}
$$

here

$\xi\left(\phi, x_{0}, h_{k, \lambda}\right)=\left[\left(r_{k, \lambda} *\left(h_{k, \lambda} \circledast e^{-j \frac{\phi}{\lambda}}\right)\right) \circledast h_{k, \lambda}^{\star}\right]\left(x_{0}\right) \cdot e^{j \frac{\phi\left(x_{0}\right)}{\lambda}}$,

$\mathcal{I}(\cdot)$ denotes the operator that takes the imaginary part of its argument, $r_{k, \lambda}=i_{k, \lambda}-o_{k, \lambda}, h_{k, \lambda}^{\star}\left(x_{0}\right)=\overline{h_{k, \lambda}\left(-x_{0}\right)}, *$ is the componentwise product and $\circledast$ is the convolution operator. Equation (5) has been obtained from [6, Equation 7] by simply exploiting the linearity of the operator $\mathcal{I}(\cdot)$. This novel formulation is much more compact and faster to compute: in fact, for an image of size $N \times N$, the gradient has now time complexity $\mathcal{O}\left(N^{2} \log \left(N^{2}\right)\right)$, instead of $\mathcal{O}\left(N^{4}\right)$ exhibited in [6].

\subsection{Choice of the step size parameter}

The stationarity of the limit points of the sequence generated by (4) is guaranteed by choosing the step size $\alpha_{m}=\alpha_{m}^{(0)} \gamma^{\ell_{m}}$, where $\gamma \in(0,1)$ and $\ell_{m}$ is the smallest natural number satisfying the Armijo condition

$$
J\left(\phi^{(m)}-\alpha_{m} \nabla J\left(\phi^{(m)}\right)\right) \leq J\left(\phi^{(m)}\right)-\beta \alpha_{m}\left\|\nabla J\left(\phi^{(m)}\right)\right\|^{2},
$$

being $\beta \in(0,1)$. The initial guess $\alpha_{m}^{(0)} \in\left[\alpha_{\min }, \alpha_{\max }\right]$, with $0<\alpha_{\min } \leq \alpha_{\max }$, is typically chosen by seeking for some second-order information of the objective function $J$ in order to improve the convergence rate of the scheme (see e.g. $[9,10])$. In our approach we used a rule recently proposed by Fletcher [7] in the case of quadratic objective functions and based on the approximation of the eigenvalues of the Hessian matrix by means of certain Ritz values related to the Lanczos process. The approach has been extended by Fletcher to nonquadratic objective functions and is based on the storage of $q$ consecutive gradients and steplengths in the matrices

$$
\begin{gathered}
G=\left[\nabla J\left(\phi^{(m-q)}\right) \ldots \nabla J\left(\phi^{(m-2)}\right) \nabla J\left(\phi^{(m-1)}\right)\right] ; \\
\Gamma=\left[\begin{array}{cccc}
\alpha_{m-q}^{-1} & & & \\
-\alpha_{m-q}^{-1} & \ddots & & \\
& \ddots & \alpha_{m-2}^{-1} & \\
& & -\alpha_{m-2}^{-1} & \alpha_{m-1}^{-1} \\
& & -\alpha_{m-1}^{-1}
\end{array}\right]
\end{gathered}
$$

If $G=Q R$ is the thin QR factorization of $G$ [11], $r=$ $Q^{T} \nabla J\left(\phi^{(m)}\right)$ and $\Xi=\left[\begin{array}{ll}R & r\end{array}\right] \Gamma R^{-1}$, then the Ritz-like approximations are computed as the eigenvalues of a symmetric and tridiagonal approximation $\widetilde{\Xi}$ of $\Xi$ defined as

$$
\widetilde{\Xi}=\operatorname{diag}(\Xi)+\operatorname{tril}(\Xi,-1)+\operatorname{tril}(\Xi,-1)^{T},
$$

where $\operatorname{diag}(\cdot)$ and $\operatorname{tril}(\cdot,-1)$ denote the diagonal and the strictly lower triangular parts of a matrix. We only remark that the matrix $\Xi$ can be obtained without the explicit computation of $Q$ by exploiting the partially extended Cholesky factorization

$$
G^{T}\left[\begin{array}{ll}
G & \left.\nabla J\left(\phi^{(m)}\right)\right]=R^{T}[R \quad r
\end{array}\right] .
$$

Once the $q$ values $\theta_{1}, \ldots, \theta_{q}$ have been obtained, their reciprocal $\theta_{1}^{-1}, \ldots, \theta_{q}^{-1}$ are used as step sizes for the following $q$ iterations, in which new gradients and step sizes will be collected to generate new matrices $G$ and $\Gamma$ and the successive group of $q$ Ritz-like values.

\section{NUMERICAL TESTS}

The proposed method has been tested on two computergenerated objects which represent the phase functions of 


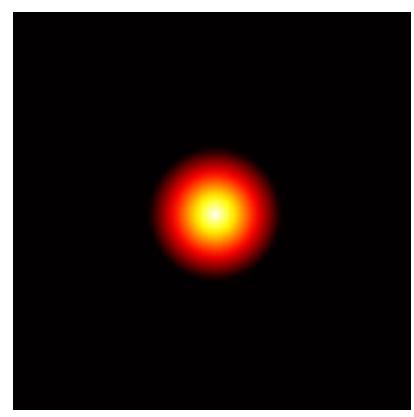

(a)

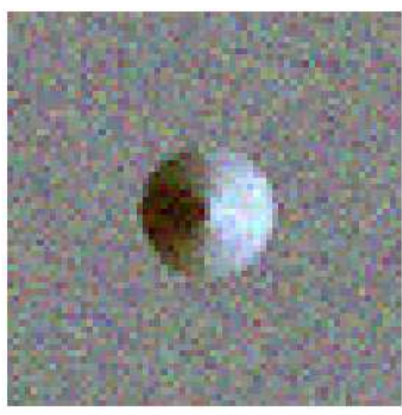

(b)

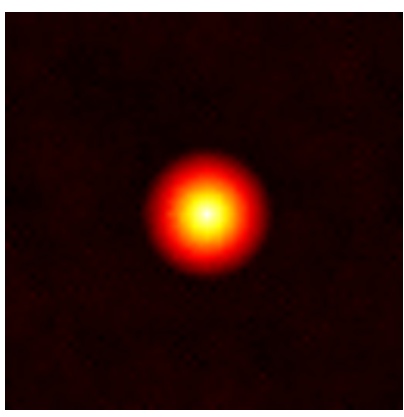

(c)

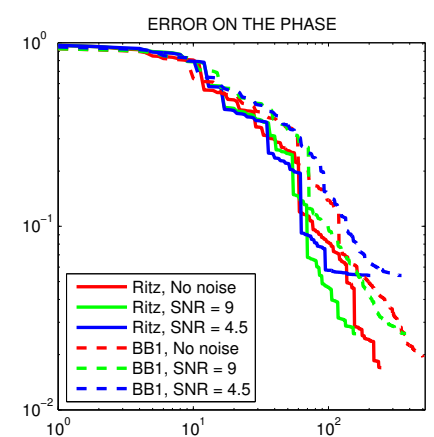

(d)

Fig. 1. Data and results for the cone object. From left to right: true object, noisy DIC color image taken at angle $\tau_{0}=0^{\circ}$ and $\mathrm{SNR}=4.5$, reconstructed phase and the relative error versus the number of iterations.
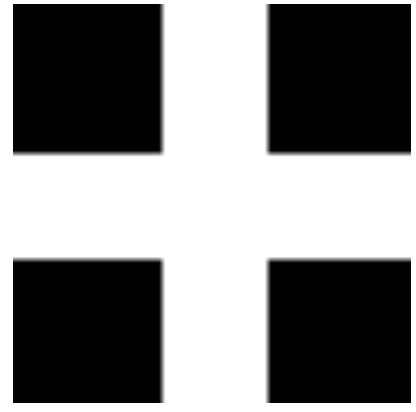

(a)

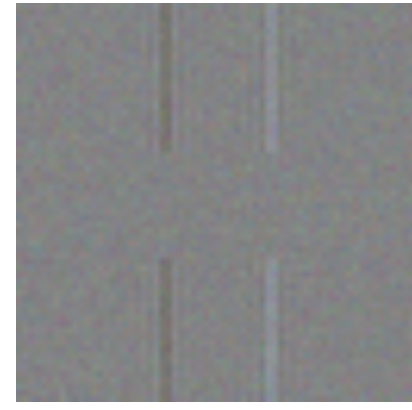

(b)

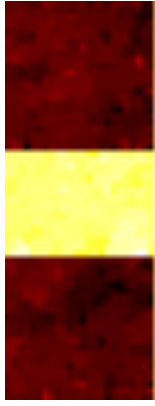

(c)

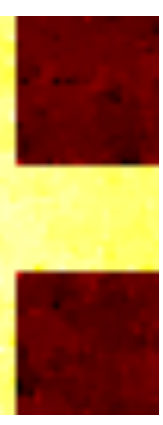

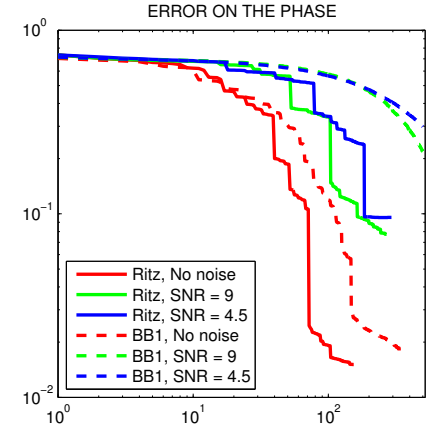

(d)

Fig. 2. Data and results for the cross object. From left to right: true object, noisy DIC color image taken at angle $\tau_{0}=0^{\circ}$ and $\mathrm{SNR}=4.5$, reconstructed phase with and the relative error versus the number of iterations.

two phantom specimens. Both objects were computed, with respect to a zero-phase background, by using relation $\phi(x)=2 \pi\left(n_{1}-n_{2}\right) t(x)$, where $n_{1}$ and $n_{2}$ are the refractive indices of the object structure and the surrounding medium, respectively, and $t(x)$ is the thickness of the object at point $x$. The first phantom is a truncated cone of radius $r=3.2 \mu \mathrm{m}$ with $n_{1}=1.33, n_{2}=1, t(x)=t_{\max }-\left(t_{\max }|x|\right) / r$ with $t_{\max }=0.7572$ and maximum value $\phi_{\max }=1.57 \mathrm{rad}$ (Figure 1(a)). The second one consists of two crossing bars, each one of width $5 \mu \mathrm{m}$, with $n_{1}=1.5039, n_{2}=1.4858, t(x) \equiv 1 \mu \mathrm{m}$ and maximum value $\phi_{\max }=0.114 \mathrm{rad}$ (Figure 2(a)).

The datasets have been generated as follows. Both the two objects described above were discretized as $64 \times 64$ images, with pixel size $0.30 \mu \mathrm{m} \times 0.30 \mu \mathrm{m}$ and an overall size of $19.2 \mu \mathrm{m} \times 19.2 \mu \mathrm{m}$. For every illumination wavelength, a DIC PSF for a $10 \times 0.3$ N.A. lens was computed as in (2), by setting $2 \Delta \theta=1.57 \mathrm{rad}$ and $\Delta x=0.68 \mu \mathrm{m}$. Two orthogonal DIC color images were generated with model (1) by rotating the three PSFs from $\tau_{0}=0^{\circ}$ to $\tau_{1}=90^{\circ}$. Finally, these images were corrupted with white Gaussian noise at a signal-to-noise (SNR) ratio equal to $9 \mathrm{~dB}$ and $4.5 \mathrm{~dB}$ (Figure 1(b) and 2(b)) respectively, being the SNR value computed from SNR $=10 \log _{10}\left(\overline{\phi^{*}} / \sigma\right)$, where $\overline{\phi^{*}}$ is the mean value of the true object and $\sigma$ is the standard deviation of noise.

The reconstruction algorithm (4) was initialized with a constant image $\phi^{(0)}=0$ and was then stopped when the norm between two successive iterates was lower than the prefixed tolerance $10^{-5}$. The step sizes were computed as described in section 2.3 every $q=4$ iterations, whereas the line-search parameters were set to $\gamma=0.5$ and $\beta=10^{-4}$. Finally, the regularization parameter and the TV smoothing parameter were manually set both equal to $10^{-2}$ for the cone and $4 \cdot 10^{-2}$ and $10^{-3}$ for the cross.

In Figure 1(c) and 2(c), we show the reconstructed phase images provided by our method with $\mathrm{SNR}=4.5$. Moreover, we compare the proposed approach to another gradient-descent method in which, at each iteration, the step size is initialized by means of the first Barzilai-Borwein (BB1) rule [9]

$$
\alpha_{m}^{B B 1}=\frac{\left(\phi^{(m)}-\phi^{(m-1)}\right)^{T}\left(\phi^{(m)}-\phi^{(m-1)}\right)}{\left(\phi^{(m)}-\phi^{(m-1)}\right)^{T}\left(\nabla J\left(\phi^{(m)}\right)-\nabla J\left(\phi^{(m-1)}\right)\right)},
$$

and then successively reduced by performing a nonmonotone linesearch, achieved by substituting the term $J\left(\phi^{(m)}\right)$ at the r.h.s. of (6) with the maximum value of the objective function $J$ in the previous $M$ iterations (in our tests we chose $M=10)$. In Figure 1(d) and 2(d), we report the recon- 
struction errors of both methods with respect to the number of iterations. In particular, the error has been computed as $\left\|\phi^{(m)}-\phi^{*}-\bar{c} \mathbf{1}\right\| /\left\|\phi^{*}\right\|$, where $\phi^{*}$ is the true object, $\mathbf{1}$ is the vector of all ones and $\bar{c}=\sum_{x \in \chi}\left[\phi^{(m)}(x)-\phi^{*}(x)\right] / N$. Unlike the usual root mean square error, this error measure takes into account the fact that, because of model (1), the true phase might be recovered only up to a real constant.

As it can be drawn from Figure 1, the reconstruction for the cone is very satisfactory even for the most noisy case, for which the relative error is below $6 \%$. Good reconstructions are also obtained for the cross: in fact, even if the estimate in Figure 2(c) is visually less satisfactory than the one for the cone, the shape is well-recovered, as well as the phase difference. In both cases, the proposed approach seems to be quite robust to noise.

Furthermore, Figure 1(d) and 2(d) show that the proposed choice for the step size outperforms significantly the standard BB1 approach, in terms of efficiency, accuracy and robustness to noise. This is particularly evident for the cross object in which, for both the considered levels of noise, BB1 recovers a coarse estimate in 500 iterations, while our approach provides an error below $10 \%$ in roughly 300 iterations. In average, the number of iterations required to achieve the estimate is reduced of nearly $50 \%$.

\section{CONCLUSIONS AND FUTURE WORK}

We presented the performance of a gradient-based optimization method applied to the problem of phase estimation from DIC images, extending the rotational-diversity approach to include the presence of polychromatic images. Numerical experience showed that: i) the method is robust with respect to the presence of high rates of noise; ii) it overcomes the assumption of no previous knowledge of the initial guess, which increases the ill-posedness of the problem; iii) it provides very good estimations in less iterations than a standard gradientdescent method; iv) the computational time is significantly reduced thanks to our convolution-based formulation.

Future work will include the analysis of the proposed method on real experimental images, where there is a limited number of observations and the sampling frequency is reduced. It is also contemplated to consider another formulation of the model by doing change of variables and designing suitable optimization methods, possibly exploiting the Fletcher step size adapted to the constrained case [12, 13].

\section{REFERENCES}

[1] Allen R. D., David G. B., , and Nomarski G., "The Zeiss-Nomarski differential interference equipment for transmitted-light microscopy," Z. Wiss. Mikrosk., vol. 69, no. 4, pp. 193-221, 1969.

[2] Preza C., Snyder D., and Conchello J. A., "Theoreti- cal development and experimental evaluation of imaging models for differential interference contrast microscopy," J. Opt. Soc. Am. A, vol. 16, no. 9, pp. 21852199, 1999.

[3] Murphy D. B., Fundamentals of light microscopy and electronic imaging, Wiley-Liss, 2001.

[4] Lin W., Yu S., and Lin S., "Accelerating phase shifting technique in quantitative differential interference contrast system for critical dimension measurement of TFT substrate," SID Int. Symp. Dig. Tec., vol. 43, no. 1, 2012.

[5] Bostan E., Froustey E., Rappaz B., Shaffer E., Sage D., and Unser M., "Phase retrieval by using transport-ofintensity equation and differential interference contrast microscopy," in Proc. 21th IEEE International Conference on Image Processing, 2014, pp. 3939-3943.

[6] Preza C., "Rotational-diversity phase estimation from differential interference contrast microscopy images," J. Opt. Soc. Am. A, vol. 17, no. 3, pp. 415-424, 2000.

[7] R. Fletcher, "A limited memory steepest descent method," Math. Program., vol. 135, no. 1-2, pp. 413436, 2012.

[8] R. Acar and C. R. Vogel, "Analysis of bounded variation penalty methods for ill-posed problems," Inverse Probl., vol. 10, no. 6, pp. 1217-1229, 1994.

[9] J. Barzilai and J. M. Borwein, "Two-point step size gradient methods," IMA J. Numer. Anal., vol. 8, no. 1, pp. 141-148, 1988.

[10] B. Zhou, L. Gao, and Y. H. Dai, "Gradient methods with adaptive step-sizes," Comput. Optim. Appl., vol. 35, no. 1, pp. 69-86, 2006.

[11] G. H. Golub and C. F. Van Loan, Matrix computations, John Hopkins University Press, Baltimore, 3rd edition, 1996.

[12] F. Porta, R. Zanella, G. Zanghirati, and L. Zanni, "Limited-memory scaled gradient projection methods for real-time image deconvolution in microscopy," Commun. Nonlinear Sci. Numer. Simul., vol. 21, no. 1-3, pp. 112-127, 2015.

[13] F. Porta, M. Prato, and L. Zanni, "A new steplength selection for scaled gradient methods with application to image deblurring," J. Sci. Comput., in press, DOI: 10.1007/s10915-015-9991-9. 